INRA Prod. Anim., 2011, 24 (4), 357-362

\title{
Sélection sur gènes et sélection assistée par marqueurs
}

J.-M. ELSEN ${ }^{1}$

${ }^{1}$ INRA, UR631, Station d'Amélioration Génétique des Animaux, F-31326 Castanet-Tolosan, France

Courriel : Jean-Michel.elsen@toulouse.inra.fr

Plusieurs plans de sélection ont valorisé des informations sur l'existence de gènes dont le polymorphisme explique une part de la variabilité des caractères, soit directement quand ces gènes étaient identifiés et les mutations causales connues, soit indirectement par leur localisation à l'aide de marqueurs génétiques. Ces sélections sur les gènes ou assistées par marqueurs se sont révélées efficaces dans un certain nombre de circonstances, mais on montré des limites qu'il faut avoir en tête au moment où la sélection génomique, basée elle aussi sur ces informations, bouleverse notre conception de la sélection.

L'essentiel des plans de sélection des animaux de ferme visait dans le passé à exploiter l'hérédité polygénique gouvernant les caractères. Toutefois, l'existence de gènes à effet visible ou majeur, puis la découverte, grâce aux techniques de marquage du génome, de régions chromosomiques impliquées dans la variation des caractères (les «QTL (Quantitative Trait Loci)») ont aussi été utilisées par les améliorateurs des populations animales. Au moment où, dans certaines filières, l'évaluation génomique vient révolutionner les pratiques de sélection, quelques enseignements peuvent être tirés de ces expériences de sélection sur des gènes identifiés ou sur des QTL.

Cet article montre dans ces deux situations la nature de l'information exploitable, les principes de son utilisation, les applications envisagées et réalisées, et les limites de ces approches.

\section{1 / Sélection sur des gènes identifiés}

\section{1 / Information disponible}

Nous nous plaçons dans les hypothèses suivantes :

Un gène à effet majeur sur un caractère d'intérêt zootechnique a été repéré. La démarche suivie pouvait être $i$ ) une observation attentive de la transmission du caractère entre générations, signant une hérédité mendélienne (observation possiblement étayée par des traitements statistiques ad hoc, de type analyse de ségrégation, e.g. Elston et Stewart 1971), ii) une approche de type QTL, dans laquelle on cherche si telle ou telle zone du génome, dont la transmission est révélée par des marqueurs génétiques, intervient dans la variation (e.g. Lander et Botstein 1989), iii) le test d'un gène candidat, c'est-à-dire connu pour intervenir dans la biologie d'un caractère, et dont on met le polymorphisme en relation avec la variabilité du trait (e.g. Barbieri et al 1995).

Ce gène a été cartographié, et la mutation causale identifiée. Cette deuxième étape est longue dans la première approche (e.g. Gènes Booroola, Lacaune, Texel), facilitée pour les QTL qui sont par construction cartographiés, avec une phase de localisation fine cependant très difficile (e.g. DGAT1), immédiate pour les gènes candidats (e.g. IgF1).

Une méthode de génotypage, permettant de connaître rapidement le génotype en ce gène majeur de tout individu, a été mise au point et est disponible à grande échelle.

\section{2 / Principes de valorisation}

Nous considérons la situation où un gène à effet important sur la variabilité d'un caractère existe. Certes, un plan de sélection, ignorant ou négligeant cette information, peut toujours être mis en œuvre et un progrès génétique sera observé. Les allèles favorables en ce gène à effet majeur seront le plus souvent favorisés par cette sélection. Cependant, d'une part les effets à long terme de cette sélection peuvent être amoindris par l'existence de ce gène (Gibson 1994) (cf. § 1.4a), d'autre part on ne tire pas profit de la possibilité de savoir rapidement le génotype des candidats à la sélection.

En effet, les techniques de génotypage sont applicables dès le plus jeune âge et dans les deux sexes, et ne nécessitent pas la mesure du phénotype de l'individu concerné. Elles ouvrent donc les possibilités de :

- réduire les intervalles de génération;

- accroître le champ de la sélection en différenciant des individus jusqu'alors non différentiables (e.g. les mâles pour un gène contrôlant un caractère exprimé uniquement chez les femelles) ;

- diminuer les coûts de mesure si le phénotypage nécessite un protocole élaboré et/ou l'abattage des animaux (e.g. composition corporelle, comportement...);

- abaisser les risques dans le cas d'une sélection sur la résistance à une maladie.

\section{3 / Applications}

Sans prétendre à l'exhaustivité, cinq types d'utilisation de l'information sur l'existence d'un gène à effet majeur peuvent être considérés, et ont été mis en application dans les populations animales.

\section{a) Elimination de tares génétiques}

Par tares génétiques nous entendrons ici des défauts majeurs (de type syndactylie, Duchesne et al (2006) ou BLAD chez les bovins, Shuster et al (1992)) ou plus quantitatifs, tels que, chez le porc, un potentiel glycolytique trop élevé dans le cas du gène RN (Milan et al 2000), ou une sensibilité au stress pour le gène de la ryanodine (Fuiji et al 1991). Une telle tare peut être en partie provoquée par un gène à effet important, voire être strictement monogénique. La démonstration de cette hérédité est obtenue en étudiant de façon ciblée les causes de ce défaut, ou dans le cadre plus large d'une recherche 
systématique de déviants (cf. l'observatoire des défauts génétiques en bovins).

Cette tare étant connue, le génotypage correspondant sera opéré dans les familles à risque (les apparentés d'un individu malade ou connu pour être porteur du défaut génétique, avec des exemples célèbres chez les taureaux), ou de façon systématique sur tous les candidats à la sélection (essentiellement les mâles pour des raisons de coût).

\section{b) Sélection d'allèles de résistance}

L'amélioration de la résistance aux maladies infectieuses par les méthodes classiques (de type «boîte noire») est difficile, le point critique étant l'obtention de phénotypes alimentant les estimations de valeurs génétiques sur lesquelles les candidats sont triés. Des mesures en ferme sont à la fois aléatoires (on ne maîtrise pas la pression infectieuse), et contradictoires avec les objectifs de l'élevage, qui se veut sain. L'organisation de tests d'infection en conditions contrôlées est le plus souvent très coûteuse car il faut travailler dans des installations protégées. Enfin, pour des maladies à durée d'incubation longue, telles que les encéphalopathies, il faut attendre des années avant d'obtenir les phénotypes. Dans quelques cas ces difficultés ont cependant été résolues (utilisation des comptages de cellules du lait pour les mammites, infestation expérimentale de parasites gastro-intestinaux chez les ovins).

Connaître un gène majeur dont certains génotypes confèrent une résistance, même partielle, à une maladie est clairement attrayant car la sélection sur le génotype en ce gène peut se faire sans mesure de phénotypes et rapidement.

L'exemple phare d'une telle sélection est trouvé chez les ovins. Le gène PrP, très polymorphe, possède un allèle («ARR») dont les porteurs résistent très bien à la tremblante, une forme spécifique d'encéphalopathie (e.g. Elsen et al 1999). Cette résistance aux formes contagieuses de la tremblante a été établie sur de très grandes séries de données. Fort de cette information, des plans d'amélioration des races ovines pour la résistance aux ESST ont été organisés dans différents pays. En France, le nombre de génotypages réalisés pour ce plan avoisine le million, avec des effets évidents sur l'évolution des fréquences génotypiques à venir sur l'incidence de la maladie (Barillet et al 2004).

c) Sélection sur un caractère non mesurable

Plus généralement, disposer d'une information sur un gène affectant la variabilité d'un caractère difficile à mesurer en conditions standards permet d'alléger la sélection et d'en accélérer les effets.

Ainsi, l'aptitude au désaisonnement de la reproduction des ovins est une caractéristique économiquement importante (les cours de la viande et les ressources fourragères dépendent de la saison) mais quasiment impossible à mesurer en ferme. Des travaux expérimentaux avaient démontré le rôle de variants du récepteur à la mélatonine sur une mesure de l'activité ovulatoire spontanée de brebis, ouvrant la porte à une sélection sur ce caractère. Si cette voie a finalement été abandonnée, une recherche plus systématique de gènes influençant le désaisonnement reste un objectif majeur pour cette espèce (Pelletier et al 2000).

\section{d) Présélection d'animaux porteurs de bons allèles}

Beaucoup de schémas de sélection incluent une phase de mise en testage sur descendance de mâles choisis en fonction de leur ascendance et caractéristiques propres. Il est possible, le cas échéant, d'inclure dans cette présélection avant testage des informations sur le génotype en un gène identifié (Larzul et al 1997).

Dans beaucoup de schémas les effectifs d'animaux candidats à la sélection sont limités, et des effets de dérive importants sont possibles, pouvant aller jusqu'à la disparition de certains allèles par le fait du hasard. Plus concrètement, si l'allèle favorable en un gène majeur est rare, il est possible qu'aucun des candidats au testage sur descendance n'en soit porteur. Dans ce cas, génotyper un réservoir de candidats et favoriser les porteurs dans le choix des candidats est une précaution utile pour assurer la diffusion de l'allèle favorable. Ce type de situation a été rencontré dans le passé chez les chèvres Saanen qui possédaient peu de copies de l'allèle A de la caséine $\alpha \mathrm{S} 1$, à effet positif sur la quantité de matière protéique du lait.

A l'inverse, quand existe un gène à fort effet sur un caractère, il est très peu efficace de mettre en compétition des mâles possédant un génotype défavorable, car il y a peu de chance qu'ils sortent favorablement indexés de l'épreuve sur descendance. C'est la situation à laquelle était confrontée la race de chèvres Alpine pour la même caséine $\alpha \mathrm{S} 1$.

\section{e) Introgression d'un allèle favorable}

Certains allèles sont spécifiques de races particulières, ou limités à un groupe racial historiquement connecté. On comprend aisément que cette situation soit le fait de mutations récentes qui n'aient pas encore diffusé dans l'ensemble de l'espèce. Cette situation facilite la mise en évidence du gène correspondant car l'exploration de la variabilité chez les descendants de parents F1 unissant cette race porteuse et une race non porteuse est assurée d'une bonne informativité.

Quand un tel variant est découvert, son introgression vers une race non porteuse est possible. L'idée est, par la réalisation d'une série suffisante de croisements en retour, de retrouver le fond polygénique de la race d'accueil sans perdre l'allèle muté. Il faut à cette fin organiser la sélection des porteurs à chaque génération, classiquement sur la base de leur performance ou après un testage sur descendance. Si le gène est cartographié et la mutation causale identifiée, le tri en est grandement simplifié.

Il est aussi possible d'accélérer le processus d'introgression en sélectionnant les animaux backcross de chaque génération sur un indicateur de leur proximité avec la race d'accueil basé sur des marqueurs du génome (Hospital et al 1992).

A titre d'exemple, on peut citer l'introgression du gène Booroola en Mérinos d'Arles et Romanov (Boomarov 1990), du gène Texel en Lacaune (Grasset et al 2009) ou le gène sans corne en Charolais.

\section{f) Accouplements valorisant un géno- type optimal}

Dans certains cas, le génotype le plus intéressant pour la production n'est pas homozygote. Il en est ainsi si l'allèle muté a un effet trop fort sur le caractère (e.g. le gène Booroola en Mérinos d'Arles, cf. Teyssier et al 2010), ou si l'homozygote correspondant n'est pas viable ou montre une fitness très détériorée (e.g., le gène $\mathrm{mh}$ provoquant l'hypertrophie musculaire en bovin, mais de fortes difficultés de vêlage pour les mères (Vissac et al 1973), ou, en ovins, le gène Inverdale qui provoque une hyperovulation à l'état hétérozygote mais une stérilité à l'état homozygote (Braw-Tal et al 1993)).

Pour gérer cette difficulté des systèmes plus ou moins contraignants ont été imaginés. Au plus simple, des lignées spécialisées sont constituées et les animaux productifs sont produits en grand nombre par croisement (e.g. la lignée INRA95 permettant l'exploitant du gène $\mathrm{mh}$ avec des mères non porteuses, Vinet 2006). Dans certaines situations (par exemple le plan proposé par 
l'INRA pour le gène Lacaune L vs. +), il faut prévoir un génotypage systématique des reproducteurs pour réaliser des accouplements $(\mathrm{L}+\mathrm{x}++$ ou $++\mathrm{x} \mathrm{L}+$ uniquement) qui ne sont pas générateurs d'animaux inutiles (LL)

\section{4 / Limites}

Les applications décrites ont toutes été réalisées à des échelles variées. Un certain nombre d'enseignements peuvent être retirés de ces expériences, montrant différentes limites à la mise en œuvre d'une sélection génique.

a) Déséquilibre de liaison généré par la sélection

Dans le cas d'une hérédité mixte, les animaux porteurs du génotype favorable au gène à effet majeur ont plus de chance d'être retenus que les autres (pour simplifier on raisonnera ici sur un locus biallélique). En conséquence, il est exercé une moindre pression de sélection sur leur valeur polygénique. De façon transitoire, on voit donc s'installer un déséquilibre de liaison entre le génotype majeur et le reste du génome, qui se traduit par un moindre progrès au long terme (une fois l'allèle favorable fixé).

Cette situation est rencontrée automatiquement quand le génotype majeur n'est pas considéré dans la décision de sélection. Elle peut être empirée si, grâce au génotypage, les génotypes favorables sont sur-sélectionnés. Donc, contrairement à l'intuition courante, qui consiste à génotyper les candidats à la sélection pour retenir en priorité les porteurs de l'allèle favorable, il faut au contraire utiliser cette information pour en freiner la fixation. On imagine plus précisément que soit optimale une sélection dynamique, avec des règles changeant au cours du temps selon l'évolution des fréquences alléliques (Dekkers et Van Arendonk 1998). Ce type de sélection, difficile à optimiser, est encore plus difficile à faire passer dans la pratique et il faut en trouver des versions quasi optimales et réalistes (Costard et al 2009).

\section{b) Génotype hétérozygote optimal}

Comme évoqué plus haut (cf. $§ 1.3 f$ ) le génotype optimal peut être hétérozygote, cette situation conduisant à la mise en place de plans de sélection et d'accouplements compliqués qui peuvent dissuader les utilisateurs. Chez les espèces faisant largement appel aux lignées et aux croisements, cette complication est admise, à preuve la très large utilisation du gène de nanisme chez la poule (Mérat et Ricard 1974) c) Allèle introgressé défavorable dans la race d'accueil

Nous ne savons pas prédire l'expression d'un allèle particulier dans une race ne le possédant pas tant qu'un processus d'introgression n'est pas mené à son terme. Diverses raisons peuvent annuler son intérêt, prouvé dans une race donneuse, quand il s'exprime dans une race receveuse :

- un effet d'échelle. A titre d'exemple, il semblerait que le gène Booroola ait un effet multiplicatif plutôt qu'additif sur le niveau d'ovulation, conduisant à des performances totalement inexploitables d'hétérozygotes B+ Romanov (5 contre 2,5 ovulations chez les ++ , alors que les valeurs sont 2 et 1 en Mérinos d'Arles);

- des effets d'épistasie défavorables avec des gènes dont les allèles diffèrent entre races donneuse et receveuse ;

- des voies métaboliques différentes selon les races, le gène introgressé étant limitant en donneuse, pas en receveuse.

Malheureusement, les résultats étant imprévisibles, il faut introgresser avant de conclure sur l'utilité de l'introgression!

\section{d) Effets défavorables sur d'autres caractères}

Plusieurs raisons font qu'un allèle montrant un effet positif pour un caractère (disons $\mathrm{C} 1$ ) peut avoir un impact négatif sur un autre caractère d'intérêt zootechnique (C2) :

- la pléiotropie, qui est rencontrée quand le même gène est impliqué dans la régulation de $\mathrm{C} 1$ et $\mathrm{C} 2$. Le gène de susceptibilité à l'halothane en porc est un exemple souvent cité. Le gène $\mathrm{RN}$ qui détériore la capacité de rétention d'eau mais est associé à une plus grande surface de noix de côtelette en fait aussi partie (Le Roy et al 2000) ;

- un déséquilibre de liaison défavorable avec un locus contrôlant C2. On pourrait presque imaginer que cette situation est inévitable quand on pense au nombre de caractères et au nombre potentiel de gènes à proximité de n'importe quel locus. L'examen positif d'effets QTL localisés sur le chromosome porteur du gène $\operatorname{PrP}$ de résistance à la tremblante illustre cette situation (Vitezica et al 2005);

- la plus grande fréquence de l'allèle favorable chez des individus génétiquement détériorateurs pour le deuxième caractère. Cette situation se rencontrera par exemple quand l'allèle a été récemment apporté à la race par la migration d'individus différant pour ce deuxième caractère ;

- un effet de type récursif par lequel le niveau de $\mathrm{C} 2$ est automatiquement modulé par celui de $\mathrm{C} 1$. Par exemple, la mortalité périnatale augmente avec la taille de portée. Le gène Booroola augmente la taille de portée et la mortalité augmente aussi. Mais des individus porteurs du gène Booroola qui auraient leur taille de portée déprimée par un quelconque procédé non génétique présenteraient une mortalité périnatale standard, ce qui ne serait pas le cas dans la situation de pléiotropie.

La diffusion large d'un allèle favorable pour un caractère devrait donc toujours être précédée d'une étude approfondie des effets de cet allèle sur une batterie de caractères importants pour l'élevage.

\section{e) Allèle de résistance contourné}

Il existe de la variabilité chez les entités pathogènes comme chez leurs hôtes. Bien plus, cette variabilité n'est pas un ensemble fermé, mais peut s'étendre par le biais des recombinaisons et mutations, génétiques ou épigénétiques. D'ailleurs, le contournement de la résistance a toujours été une préoccupation majeure en vaccinologie et antibiothérapie. La sélection pour la résistance aux maladies infectieuses n'échappe pas à cette difficulté.

Qu'il préexiste à de très faibles fréquences et émerge avec la disparition de versions vaincues par la résistance de l'hôte, ou qu'il apparaisse par mutation, un variant de l'agent pathogène capable d'attaquer les animaux sélectionnés pour leur résistance supposée remet totalement en cause cette sélection et doit donc être traqué en permanence. L'exemple des encéphalopathies ovines pour lesquelles une diversité a été progressivement démontrée, avec une forme atypique, très peu transmissible, qui ne se développe pas chez les porteurs d'un des allèles de forte sensibilité à la tremblante classique en est une bonne démonstration (Moreno et al 2007). Sans remettre vraiment en cause le plan français de sélection pour la résistance à cette pathologie (l'allèle ARR de résistance à la tremblante classique est neutre pour l'atypique), cette observation tempère fortement l'objectif du «tout ARR» et appuie la nécessité d'un suivi permanent de la résistance et d'une conservation de la variabilité génétique.

\section{2 / Sélection assistée par marqueurs}

\section{1 / Information disponible}

Nous considérons maintenant le cas où une exploration systématique du génome a été réalisée à l'aide de marqueurs (type microsatellites ou SNP), le 
plus souvent dans des familles suffisamment grandes pour que la transmission de segments chromosomiques des parents vers les descendants puisse être tracée avec précision. A la suite de cette exploration, plusieurs zones ont été repérées, porteuses de QTL. Ces zones sont plus ou moins larges en fonction de l'information disponible. Jusqu'à une date récente on distinguait une première étape de détection, basée sur la mise en relation, pour chaque segment du génome, des valeurs phénotypique et de la transmission de ce segment, et une deuxième étape de cartographie fine visant à préciser la localisation des QTL par une augmentation du nombre de méioses et de marqueurs et par l'utilisation du déséquilibre de liaison observable entre deux localisations le plus souvent très proches. Avec le développement des puces à SNP, on envisage maintenant de court-circuiter la phase de détection et d'aller directement vers des approches globales de l'association.

A partir de ces informations, il est possible de construire des outils de génotypage des QTL basés sur les informations de marqueurs localisés à leur proximité. Dans le cas d'une cartographie lâche (en général une précision de localisation de 10 à 30 centiMorgans $(\mathrm{cM})$, obtenue après la première étape de détection) l'information n'est valable qu'intra famille et doit sans cesse être réétalonnée. Dans le cas d'une cartographie fine (de l'ordre du cM, après une analyse du déséquilibre de liaison), l'information est valable entre familles, et assez stable.

\section{2 / Principes de valorisation}

La sélection assistée par marqueurs possède a priori les mêmes attraits que la sélection génique, puisqu'elle permet aussi de réduire les intervalles de génération, accroître le champ de la sélection en différenciant des individus jusqu'alors non différentiables, diminuer les coûts de mesure et réduire les risques. Comme pour la sélection génique, il s'agira de génotyper des candidats à la sélection le plus rapidement possible, et d'organiser un tri et des accouplements basés à la fois sur l'information de ce génotypage et sur les données phénotypiques et pedigree classiques.

Cependant, elle en diffère de deux façons :

- les données ne portent pas sur les génotypes actifs mais sur des marqueurs. Cette association est instable. Ne pas considérer cette instabilité se traduirait par une baisse progressive de l'efficacité de la sélection. Comme évoqué plus haut, la fréquence nécessaire du réétalonnage dépend de la qualité de l'information ;
- en principe (sans doute beaucoup moins en pratique) les informations QTL sont polygéniques : à l'issue d'une expérience de détection/localisation, on ne connaît pas une mais plusieurs zones porteuses de QTL, qui, cumulées, expliquent une part importante de la variabilité. On est alors tenté de rassembler cette information dans un indicateur unique, une estimation de valeur génétique assistée par marqueur (MAEBV), qui combine toute l'information disponible, de nature génotypique et phénotypique, et de trier les candidats à la reproduction sur cette MAEBV.

\section{3 / Applications}

En fonction de la précision de l'information disponible on distingue deux types de sélection assistée par marqueurs.

\section{a) SAM exploitant la liaison géné- tique ("SAM1»)}

Le prototype de cette sélection est un protocole en deux temps :

- dans le cadre d'un test de la descendance, pour chaque segment chromosomique, les effets des deux versions grand parentales qu'un père transmet à sa descendance sont contrastés, et des segments significatifs repérés ;

- les fils produits de ce père sont génotypés pour les marqueurs des segments significatifs et la valeur aux QTL de ces fils estimée (et potentiellement combinée avec tout autres informations disponibles). Les meilleurs sont présélectionnés comme candidats à la mise en testage, et le cycle reprend.

Les «BLUPeurs», concernés par l'évaluation génétique, se sont emparés de la question et ont mis en place un outillage pour estimer les MAEBV au mieux et à grande échelle. Fernando et Grossman (1989) ont été les précurseurs de la démarche.

Les premières évaluations de l'intérêt de la SAM (Ruane et Colleau 1995) étaient très défavorables. Si l'on observait un gain important sur les QTL euxmêmes, et si l'efficacité à très court terme était bonne, la SAM perdait rapidement de sa valeur et, après peu de générations, donnait de moins bons résultats que la sélection classique. Cette évaluation n'envisageait pas de changement de plan de sélection et se limitait à l'amélioration d'un caractère que les procédures standards de sélection étaient capables d'améliorer efficacement.

La SAM permet de réduire les intervalles de générations en donnant des informations sur la valeur des individus dès leur plus jeune âge. Appliquée à un schéma de type MOET (Multiple
Ovulation and Embryo Transfer) dans lequel les mâles sont utilisés très rapidement, la SAM est efficace, avec un gain de 10 à $20 \%$ sur les schémas sans marqueur (Ruane et Colleau 1996). De la même façon, elle doit permettre de sélectionner des caractères difficiles à améliorer par les techniques classiques.

En pratique, une SAM de type 1 a été mise en place en France sur les bovins laitiers (Boichard et al 2002). Des dizaines de milliers d'individus ont été génotypés pour 43 microsatellites marquant 14 QTL. Parmi les arguments développés en faveur de cette organisation, la réduction possible, de 8 à $33 \%$ selon les caractères, du nombre de taureaux à tester pour obtenir le même progrès génétique qu'en l'absence d'informations moléculaires, a été mise en avant.

Dans un article de bilan publié en 2007, Fritz et al (2007) ont montré que la mise en route de la SAM en bovins laitiers en France entre 2001 et 2007 a permis d'augmenter la précision des index des jeunes animaux : évolution moyenne des CD de 0,18 pour la fertilité ou 0,33 pour la quantité de lait en sélection classique (sans marqueurs) à respectivement 0,29 et 0,44 en SAM.

\section{b) SAM exploitant le déséquilibre de} liaison ( «SAM2»)

A l'issue d'un protocole de localisation fine, ou d'un protocole unique basé sur un grand nombre de marqueurs SNP, des haplotypes marqueurs caractérisant des petits segments chromosomiques portant des QTL sont inventoriés. Un haplotype est associé au même allèle QTL dans toutes les familles. Compte tenu de la faible taille de ces segments, les évènements de recombinaison sont rares, et une double recombinaison, invisible, quasiment impossible. Dans ces conditions, la SAM2 est très proche d'une sélection (multi)-génique (Guillaume et al 2008).

$\mathrm{Au}$ moment où la SAM2 pouvait démarrer dans les populations d'élevage françaises, la sélection génomique a émergé. La plupart des opérateurs ont choisi de se lancer directement dans ce type de sélection. En France, compte tenu de la richesse des informations accumulées, le choix a été fait de combiner SAM2 et sélection génomique (Guillaume 2011).

\section{4 / Limites}

a) La sélection assistée par marqueurs partage les limites de la sélection génique

Les difficultés liées à l'apparition d'un déséquilibre de liaison avec le fond 
polygénique sont aussi présentes dans la SAM. L'évolution défavorable de l'efficacité de la SAM au cours des générations est sans doute en partie due à ce phénomène (les recombinaisons diminuant le pouvoir prédictif des marqueurs et la fixation des allèles QTL étant aussi en cause). Il ne semble pas que cette question ait été traitée de façon satisfaisante, laissant un pan de recherche possible. Toutefois, plusieurs QTL du même caractère étant simultanément sélectionnés, l'apparition d'un déséquilibre de liaison défavorable entre les allèles positifs de ces QTL et le fond polygénique est limitée.

Les algorithmes imaginés par les spécialistes de l'évaluation génétique visent essentiellement à exploiter l'additivité des QTL. Les situations où les hétérozygotes sont l'optimum, ainsi que l'existence d'interactions entre QTL, sont très difficiles à prendre en compte.

\section{b) La nécessité du réétalonnage}

Evoquée plusieurs fois dans cet article la nécessité du réétalonnage est une contrainte importante, financière et d'organisation, qui existera aussi avec la sélection génomique. L'exemple bovin laitier, qui repose sur des ressources en information et budgétaires considérables par rapport à d'autres filières, ne saurait être généralisé sans précaution.

\section{Conclusion}

Il est possible que la sélection génomique prenne le pas sur toute autre forme de sélection dans les prochaines années. Dans chaque espèce, ceci dépendra du coût relatif du génotypage.

Nombre des applications qui ont été décrites pour la sélection génique, ainsi que ses limites, sont transposables à cette situation nouvelle. Les possibilités de diminuer les intervalles de génération et de sélectionner mâles et femelles sur des marqueurs de caractères difficiles à phénotyper sont communes aux deux approches. Les problèmes liés à l'apparition d'associations négatives entre locus, à la pléiotropie défavorable ou à l'existence de SNP en déséquilibre de liaison l'un agissant positivement, l'autre négativement, sur deux caractères, seront aussi rencontrés.

Par ailleurs, la sélection génique restera une approche importante pour les gènes à effet visible ou majeur, notamment pour des caractères tels que la résistance aux maladies. Il faudra donc combiner les deux types de sélection. On peut d'ailleurs étendre cette conclusion aux cas des QTL non (encore) identifiés mais très bien localisés, grâce au déséquilibre de liaison. La sélection assistée par marqueur de deuxième génération («SAM2») doit être revue en association avec la sélection génomique. Le dispositif français de sélection des bovins laitiers donne un exemple d'une telle combinaison.

La construction de génotypes, l'introgression multigénique, sont autant de démarches sortant du modèle linéaire dominant que les évaluateurs ont su adapter aux nouvelles donnes de la génomique. Des approches adaptées devront être élaborées.

\section{Références}

Barbieri E., Manfredi E., Elsen J.M., Ricordeau G., Bouillon J., Grosclaude F., Mahé M.F., Bibé B., 1995. Influence du locus de la caséine $\alpha_{\mathrm{S} 1}$ sur les performances laitières et les paramètres génétiques des chèvres de race Alpine. Genet. Sel. Evol., 27, 437-450.

Barillet F., Palhière I., Astruc J.M., Brochard M., Baelden M., Aguerre X., Fidele F., Arranz J.M., Belloc J.P., Briois M., Frégeat G., Soulas C., Andreoletti O., Corbière F., Schelcher F.. 2004. Le programme français d'éradication de la tremblante du cheptel ovin fondé sur l'utilisation de la génétique. In : Numéro Hors-série, Encéphalopathies Spongiformes transmissibles animales. Elsen J.M. (Ed). INRA Prod. Anim., 87-100.

Boichard D., Fritz S., Rossignol M.N., Boscher M.Y., Malafosse A., Colleau J.J., 2002. Implementation of Marker-Assisted Selection in French dairy cattle. $7^{\text {th }}$ World Congr. Genet. Appl. Livest. Prod., August 1923, Montpellier, France, Comm. 22-03.

Boomarov O., 1990. The Boorrola gene in France. Major Gene for reproduction in sheep. $2^{\text {nd }}$ Int. Workshop. Toulouse, France, July 16$18,47-51$.

Braw-Tal R., McNatty K.P., Smith P., Heath D.A., Hudson N.L., Phillips D.J., McLeod B.J., Davis G.H., 1993. Ovaries of ewes homozygous for the X-linked inverdale gene (FecX') are devoid of secondary and tertiary follicles but contain many abnormal structures. Biol. Reprod., 49, 895-907.

Costard A.D., Vitezica Z.G., Moreno C.R., Elsen J.M., 2009. A dynamic deterministic model to optimize a multiple-trait selection scheme. J. Anim. Sci., 87, 885-894.

Dekkers J.C.M.,Van Arendonk J.A.M.,1998. Utilizing selection for quantitative traits with information on an identified locus in outbred populations. Genet. Res., 71, 257-275.

Duchesne A., Gautier M., Chadi S., Grohs C., Floriot S., Gallard Y., Caste G., Ducos A., Eggen A., 2006. Identification of a doublet missense substitution in the bovine LRP4 gene as a candidate causal mutation for syndactyly in Holstein cattle. Genomics, 88, 610-621.

Elsen J.M., Amigues Y., Schelcher F., Ducrocq V., Andreolletti O., Eychenne F., Vu Tien Khang J., Poivey J.P., Lantier F., Laplanche J., 1999. Genetic susceptibility and transmission factors in scrapie: detailed analysis of an epidemic in a closed flock of Romanov. Arch. Virol., 144, 431-445.

Elston R.C., Stewart J., 1971. A general model for the genetic analysis of pedigree data. Hum. Hered., 21, 523-542.

Fernando R.L., Grossman M., 1989. Marker assisted selection using best linear unbiased prediction. Genet. Sel. Evol., 21, 467-477.

Fritz S., Druet T., Guillaume F., Malafosse A., Bosher M.Y., Eggen A., Gautier M., Colleau J.J., Boichard D., 2007. Bilan du programme de Sélection Assistée par Marqueurs dans les trois principales races bovines laitières françaises et perspectives d'évolution. Renc. Rech. Rum., 129-132.

Fujii J., Otsu K., Zorzato F., de Leon S., Khanna V.K., Weiler J.E., O'Brien P.J., MacLennan D.H., 1991. Identification of a mutation in porcine ryanodine receptor associated with malignant hyperthermia. Science, 253, 448-451.

Gibson J.P., 1994. Short term gain at the expense of long term response with selection of identified loci. $5^{\text {th }}$ World Congr. Genet. Appl.
Livest. Prod., Guelph, Canada, August, 201204.

Grasset D., Bouix J., Bibé B., Leveziel H., Georges M., Laville E., 2009. Le gène d'hypertrophie musculaire du «Texel Belge» : identification, impact, introgression. Renc. Rech. Rum., 16.

Guillaume F, Fritz S., Boichard D., Druet T, 2008. Estimation by simulation of the efficiency of the French marker-assisted selection program in dairy cattle. Genet. Sel. Evol., 40, 91102.

Guillaume F., Boichard D., Ducrocq V., Fritz S., 2011 Utilisation de la sélection génomique chez les bovins laitiers. In : Numéro spécial, Amélioration génétique. Mulsant P., Bodin L., Coudurier B., Deretz S., Le Roy P., Quillet E., Perez J.M. (Eds). INRA Prod. Anim., 24, 363-368.

Hospital F., Chevalet C., Mulsant P. 1992. Using markers in gene introgression breeding programs. Genetics, 132, 1199-1210.

Lander E.S., Botstein D., 1989. Mapping mendelian factors underlying quantitative traits using RFLP linkage maps. Genetics, 121, 185199.

Larzul C., Manfredi E., Elsen J.M., 1997. Potential gain from including major gene information in breeding value estimation. Genet. Sel. Evol., 29, 161-184.

Le Roy P., Elsen J.M., Caritez J.C., Talmant A., Juin H., Sellier P., Monin G., 2000. Comparison between the three porcine $\mathrm{RN}$ genotypes for growth, carcass composition and meat quality traits. Genet. Sel. Evol., 32, 165186. 
Mérat P., Ricard F.H., 1974. Etude d'un gène de nanisme lié au sexe chez la poule: importance de l'état d'engraissement et gain de poids chez l'adulte. Ann. Génét. Sél. Anim., 6, 211217.

Milan D., Jeon J.T., Looft C., Amarger V., Robic A., Thelander M., Rogel-Gaillard C., Paul S., Iannuccelli N., Rask L., Ronne H., Lundstrom K., Reinsch N., Gellin J., Kalm E., Le Roy P., Chardon P., Andersson L.A., 2000. A mutation in PRKAG3 associated with excess glycogen content in pig skeletal muscle. Science, 288, 1248-1251.

Moreno C.R., Moazami-Goudarzi K., Laurent P., Cazeau G., Andreoletti O., Chadi S. Elsen J.M., Calavas D., 2007. Which PrP haplotypes in a French sheep population are the most susceptible to atypical scrapie? Arch. Virol., 152, 1229-1232.

Pelletier J., Bodin L., Hanocq E., Malpaux B., Teyssier J., Thimonier J., Chemineau P.,
2000. Association between expression of reproductive seasonality and alleles of the gene Met1A receptor in the ewe. Biol. Reprod., 62, 1096-1101.

Ruane J., Colleau J.J., 1995. Marker assisted selection for genetic improvement of anima populations when a single QTL is marked. Genet. Res., 66, 78-83.

Ruane J., Colleau J.J., 1996. Marker-assisted selection for a sex-limited character in a nucleus breeding population. J. Dairy Sci., 79, 1666-1667.

Shuster D.E., Kehrli M.E. Jr, Ackermann M.R., Gilbert R.O., 1992. Identification an prevalence of a genetic defect that causes leukocyte adhesion deficiency in Holstein cattle Proc. Natl. Acad. Sci. USA, 89, 9225-9229.

Teyssier J., Bodin L., Maton C., Bouquet P.M. Elsen, J.M. 2010. Biological and economic consequences of introgression of the FecB gene into the French Mérinos d'Arles sheep. Proc. Helen Newton Turner Memorial Int. Workshop, Pune, Maharashtra, India, 10-12 November, 2008. ACIAR Proc. Series 2009, $133,128-134$

Vinet A., Ménissier F., Caste G., Astruc S., Renand G., 2006. Bilan génétique de 15 ans de sélection du troupeau souche INRA95. Renc. Rech. Rum., 205-208.

Vissac B., Ménissier F., Perreau B., 1973. Etude du caractère culard. VII. Croissance et musculature des femelles; déséquilibre morphologique au vêlage. Ann. Genet. Sel. Anim., 5, 23-28

Vitezica Z.G., Moreno C.R., Bouix J., Barillet F., Perret G., Elsen J.M., 2005. A study on associations between PrP genotypes and meat traits in French sheep breeds. Anim. Sci. $81,325-330$

\section{Résumé}

Jusqu'à une date très récente (l'émergence de la sélection génomique), l'essentiel des plans de sélection était basé sur l'évaluation des valeurs génétiques à partir des informations phénotypiques et de pedigree. Cependant, ont aussi été mis en place plusieurs exemples réussis de sélection valorisant la connaissance de gènes à effets importants sur les caractères ou utilisant l'aide de marqueurs moléculaires (la «Sélection Assistée par Marqueurs»). Ces exemples sont riches en enseignements pour l'avenir de la sélection. Les applications furent variées, tant en termes de type de caractères (reproduction, qualité de carcasse, résistance aux maladies...) que d'organisation (éliminations d'allèles défavorables, introgression, accouplements ciblés...). Les limites, qui sont détaillées, de ces plans de sélection sur gènes ou marqueurs, sont autant de contraintes qu'il faudra intégrer dans l'organisation de la sélection génomique.

\section{Abstract}

\section{Gene and Marker Assisted Selection}

Until recently (when genomic selection started at a large scale), most of the selection plans were based on breeding value estimations from phenotypic and pedigree information. Nevertheless, a number of successful breeding schemes were also organized, using information on genes displaying large effects on the traits or using genomic markers ("Marker Assisted Selection"). These examples carry useful information about the future of selection. Realizations were polymorphic, both in terms of traits (reproduction, carcass quality, disease resistance...) and organization (counter selection of unfavorable alleles, between breeds introgression, mate selection...). Limits of these schemes, which are detailed in this paper, are constraints which will have to be considered in the future genomic selection organization.

ELSEN J.M., 2011. Sélection sur gènes et sélection assistée par marqueurs. In : Numéro spécial, Amélioration génétique. Mulsant P., Bodin L., Coudurier B., Deretz S., Le Roy P., Quillet E., Perez J.M. (Eds). INRA Prod. Anim., 24, $357-362$. 